\title{
Uso do tempo e atividades significativas de adultos submetidos ao transplante de células- tronco hematopoéticas
}

Uso del tiempo y actividades significativas de adultos que experimentan transplante de células madre hematopoyéticas

Use of time and meaningful activities in adults undergoing hematopoietic stem cell transplantation

\author{
Natália Moreno Ulrich ${ }^{1}$ \\ Yara Batista da Luz ${ }^{2}$ \\ Dayane Regina dos Santos ${ }^{3}$
}

Recibido: 23 de febrero 2018 • Enviado para modificación: 24 de abril 2018 • Aceptado: 30 de junio 2018

Ulrich, N.M., da Luz, Y.B. \& dos Santos, D.R. (2018). Uso do tempo e atividades significativas de adultos submetidos ao transplante de células-tronco hematopoéticas. Revista Ocupación Humana, 18(1), 7-20.

\section{RESUMO}

Este trabalho objetiva avaliar o tempo dedicado às atividades cotidianas, sua significação e suas alterações durante o processo de transplante de células-tronco hematopoéticas em adultos. Trata-se de uma pesquisa descritiva, exploratória com abordagem quantitativa. A coleta de dados foi realizada na enfermaria do Serviço de Transplante de Medula Óssea de um hospital universitário do sul do Brasil. Foram utilizados questionário sociodemográfico e o Diário de Atividades em dois momentos, com pacientes internados para realização de transplante de células-tronco hematopoéticas. Os dados foram verificados por meio de análise estatística descritiva simples. A amostra foi de 18 participantes. Atividades como banho/ higiene íntima/uso do vaso, vestuário, alimentação e sono, continuaram sendo realizadas

\footnotetext{
${ }^{1}$ Terapeuta ocupacional. Residente Programa de Residência Integrada Multiprofissional em Atenção Hospitalar em Oncologia e Hematologia, Complexo Hospital de Clínicas, Universidade Federal do Paraná. Curitiba, Brasil. namoreno.ulrich@hotmail.com iD https://orcid.org/0000-0003-4465-165X

${ }^{2}$ Terapeuta Ocupacional. Residente Programa de Residência Integrada Multiprofissional em Atenção Hospitalar em Oncologia e Hematologia, Complexo Hospital de Clínicas, Universidade Federal do Paraná. Curitiba, Brasil. yarabluz@gmail.com iD https://orcid.org/0000-0002-3432-1887

${ }^{3}$ Terapeuta Ocupacional. Magíster en Educación Física. Serviço de Transplante de Medula Óssea, Complexo Hospital de Clínicas, Universidade Federal do Paraná. Docente, Departamento de Terapia Ocupacional, Universidade Federal do Paraná. Curitiba, Brasil. dayterapeuta@gmail.com

ID https://orcid.org/0000-0001-6618-8526
} 
por $100 \%$ dos participantes durante a internação. Houve aumento do tempo para realização de tratamentos médicos e terapia, bem como para descansar e receber visitas. Atividades como locomoção/deslocamento deixaram de ser realizadas, enquanto apareceram comportamentos como chorar e ficar chateado/deprimido. O trabalho remunerado se manteve significativo nas duas entrevistas. A alimentação apresentou queda na satisfação, porém manteve-se significativa. Pode-se concluir que diversas atividades sofrem alterações durante o transplante, demonstrando a importância da presença do terapeuta ocupacional na equipe multiprofissional para auxiliar na ressignificação do cotidiano e na retomada de atividades significativas ao longo desse processo.

\section{PALAVRAS CHAVE}

transplante de células-tronco hematopoéticas, Terapia Ocupacional, hospitalização

\section{RESUMEN}

Este estudio tiene como objetivo evaluar el tiempo dedicado a actividades cotidianas, su significado y alteraciones durante el proceso de trasplante de células madre hematopoyéticas en adultos. Es una investigación descriptiva, exploratoria, con abordaje cuantitativo. Los datos se recolectaron en la enfermería del Servicio de Trasplante de Médula Ósea de un hospital universitario en el sur de Brasil. Fueron utilizados un cuestionario sociodemográfico y el Diario de Actividades en dos momentos, con pacientes internados para la realización del transplante. Se realizó análisis estadístico descriptivo simple de los datos. La muestra fue de 18 participantes. Actividades como baño/higiene íntima/uso de sanitario, vestuario, alimentación y sueño se mantuvieron en todos los participantes. Aumentó el tiempo para la realización de tratamientos médicos y terapia, descanso y recibir visitas. Actividades como locomoción/desplazamiento se dejaron de realizar, aparecieron comportamientos como Ilorar y enojarse/deprimirse. El trabajo remunerado se mantuvo significativo en las dos entrevistas. La alimentación presentó una disminución en la satisfacción, pero siguió siendo significativa. Se puede concluir que diversas actividades sufren alteraciones durante el transplante, demostrando la importancia del terapeuta ocupacional en el equipo multidisciplinar para intervenir en la resignificación de lo cotidiano y en retomar actividades significativas a lo largo del proceso.

\section{PALABRAS CLAVE}

trasplante de células madre hematopoyéticas, Terapia Ocupacional, hospitalización

\section{ABSTRACT}

This study aims to evaluate the time dedicated to daily life activities, their meaning, and their changes in adults undergoing a hematopoietic stem cell transplantation. It is a descriptive exploratory study with a quantitative approach. Data was collected at the ward of the Bone Marrow Transplantation Unit of a university hospital located in southern Brazil. A socio-demographic questionnaire and an Activity Log were used in two time points with patients hospitalized for the transplantation. Data was analyzed using simple descriptive statistics. The sample comprised 18 participants. Activities such as bathing/personal hygiene/use of toilet, clothing, feeding and sleep were still being performed by $100 \%$ of participants during their hospitalization. There was an increase of time for performing medical treatments and therapy, rest and visits. Activities such as mobility/movement were no 
longer performed, and behaviors such as crying and feeling angry/depressed were present. Paid work was shown to be significant in both interviews. There was a decreased satisfaction when feeding, although it kept being significant. In conclusion, several activities are susceptible to change during the transplant, which shows the importance of an occupational therapist as part of the interprofessional team, to intervene in the resignification of the daily living and to resume meaningful activities throughout this process.

\section{KEY WORDS}

hematopoietic stem cell transplantation, Occupational Therapy, hospitalization

\section{Introdução}

O transplante de células-tronco hematopoéticas tem como objetivo enxertar a célula progenitora hematopoética, a fim de solucionar defeitos quantitativos ou qualitativos da medula óssea. Este tipo de transplante é utilizado no tratamento de doenças hematológicas malignas e não-malignas, imunodeficiências, erros inatos de metabolismos e tumores sólidos (Pasquini, 2004).

O processo de transplante envolve diversos procedimentos e uma longa hospitalização, que engloba o período de condicionamento. Nesta fase, são realizadas altas doses de quimioterapia, acompanhadas (ou não) de radioterapia, além da infusão das células hematopoéticas e a recuperação da função hematopoética, a chamada "pega medular" (Oliveira-Cardoso, Voltarelli, Santos \& Mastropietro, 2005).

Nesta etapa, são observadas as principais complicações classificadas como agudas, por ocorrerem antes dos 100 dias pós-transplante. Dentre elas, podemos citar as relacionadas: à toxicidade gastrointestinal, como náuseas, vômitos, mucosite, xerostomia e diarreia; às complicações hematológicas, dermatológi- cas, renais, neurológicas, pulmonares e cardíacas; às infecções; e as relacionadas à Doença do Enxerto contra o Hospedeiro (Guedes et al., 2004).

Após a alta, o paciente continua sendo acompanhado em regime ambulatorial, até que complete as medicações necessárias e retome, gradualmente, suas atividades. Sendo assim, os pacientes que passam pelo transplante de células-tronco hematopoéticas apresentam uma necessidade de construir ou reconstruir seu cotidiano, visto que muitas de suas perspectivas e hábitos são alterados como, por exemplo, a alimentação e higiene (Bergkvist, Fossum, Johansson, Mattsson \& Larsen, 2018). Por outro lado, as principais rupturas no cotidiano do sujeito podem ser observadas durante a internação, no qual passa por diversas privações, alterando, assim, suas ocupações e a maneira como utiliza seu tempo.

O tempo é um recurso distribuído igualmente entre todas as pessoas, mas pode ser usado de diferentes maneiras. Desta forma, observa-se a importante correlação entre o fazer, as ocupações e o uso do tempo, visto que a forma como o indivíduo organiza e distribui seu tempo, pode afetar o seu bem-estar econômico e social e também impactar 
sobre sua família e a comunidade (Cavalcanti, Paulo \& Hany 2010). Assim, o uso do tempo pode ser considerado como um indicador de competência comportamental e bem-estar, sempre respeitando e levando em consideração as limitações funcionais (Doimo, Derntl \& Lago, 2008).

O terapeuta ocupacional, por meio das particularidades de cada indivíduo (seus recursos, tempo e ritmo), pode auxiliá-lo a construir seu novo cotidiano e tornar sua produção particular, singular e única. Os pacientes submetidos ao transplante de células-tronco hematopoéticas necessitam do suporte do terapeuta ocupacional ao longo de todo processo de reconstrução. Este profissional auxiliará a estabelecer metas terapêuticas praticáveis e graduais, respeitando as peculiaridades e limitações dos pacientes, e, especialmente, que os ajudem a potencializar as transformações da experiência vivida (Mastropietro, 2003; Braveman, Hunter, Nicholson, Arbesman \& Lieberman, 2017).

Os terapeutas ocupacionais têm como premissa que as atividades são inerentes aos indivíduos e a realização delas satisfaz necessidades e desejos. Entretanto, mais importante do que o ato de realizar essas atividades é o significado de cada uma para os indivíduos que as executam. Este significado é influenciado por gostos, história pessoal e a crença de que se está fazendo o correto naquele momento. Este significado envolve uma interpretação pessoal, podendo mudar de uma pessoa para outra. Assim, na perspectiva da ciência ocupacional, a ocupação é a realização de qualquer tipo de atividade com um propósito e sentido (Gomez-Lillo, 2003).
Durante a internação para realização do transplante de células-tronco hematopoéticas, o paciente é submetido a uma série de procedimentos, que podem afetar, de maneira significativa, suas ocupações devido às implicações da própria terapêutica.

As ocupações são elementos importantes para a construção da identidade e senso de competência do sujeito, além de ter significado especial e valor para esta pessoa (AOTA, 2015). O termo ocupação, segundo a American Occupational Therapy AssociationAOTA (2015), refere-se às "atividades de vida diária nas quais as pessoas se envolvem" (p.6). Ocupações adaptam-se a um determinado contexto (cultural, pessoal, físico, social, temporal ou virtual) e sofrem influências por diversos motivos, que podem ser fatores do cliente (valores e crenças), suas habilidades de desempenho (habilidades motoras e sociais por exemplo) e padrões de desempenho (como hábitos, rotinas e papéis).

Desta maneira, a presença do terapeuta ocupacional na equipe multiprofissional é de extrema importância, pois através de sua atuação poderá auxiliar o paciente a retomar papéis, atividades significativas e buscar opções adaptadas a sua nova situação, sempre de acordo com sua condição clínica. Para que a intervenção terapêutica ocupacional tenha seus objetivos atingidos, é importante a realização de uma avaliação completa, a fim de identificar quais ocupações encontram-se prejudicadas.

A temática relacionada ao uso do tempo e das atividades significativas vem sendo estudada por vários pesquisadores (Doimo, Derntl \& Lago, 2008; Fon- 
toura, Pinheiro, Galiza \& Vasconcelos, 2010; Nunes, 2015; Paganelli, 2015). Entretanto, não foi encontrado nenhum trabalho que abordasse, especificamente, a população submetida ao transplante de células-tronco hematopoéticas. Estas pesquisas na área têm como objetivo identificar a maneira como é utilizado o tempo, a fim de haver investimentos em políticas públicas e intervenções terapêuticas ocupacionais que tragam melhorias para a população.

Em relação ao transplante de células-tronco hematopoéticas, os estudos encontrados abordam as mudanças ocorridas durante o processo, como os papéis ocupacionais (Dias, Mastropietro, Oliveira-Cardoso \& De Carlo, 2012), a atuação do terapeuta ocupacional neste cenário (Paulino, Paulino, Puga \& Bianchin, 2007) e as situações estressantes vivenciadas pelos pacientes (Santos, Camargo, Santos \& Lolatto, 2017). Todavia, nenhum estudo abordou, especificamente, a relação do uso do tempo e a significação ou satisfação das atividades que sofreram alterações nesse processo, justificando-se, assim, a realização do presente estudo.

Diante do exposto, este estudo tem como objetivo avaliar o tempo dedicado às atividades cotidianas e sua significação, bem como suas alterações nos pacientes em processo de transplante de células-tronco hematopoéticas.

\section{Metodologia}

Trata-se de uma pesquisa descritiva, exploratória, com abordagem quantitativa.

A coleta de dados foi realizada na enfermaria do Serviço de Transplante de
Medula Óssea de um hospital universitário do sul do Brasil, no período de fevereiro a setembro de 2017.

A coleta foi dividida em duas etapas, sendo a primeira até 72 horas após a internação, e a segunda no momento que o paciente possuía condições de alta clínica, aproximadamente após um mês de internação. Inicialmente, o participante respondeu uma entrevista semiestruturada, com dados sociodemográficos, criados pelas pesquisadoras, a fim de caracterizar a população. Além disso, o "Diário de Atividades" foi utilizado tanto na primeira entrevista, quanto na segunda. No primeiro momento, o participante era instruído a responder tomando como base um dos últimos sete dias antes da hospitalização, o qual tenha considerado um dia com rotina de atividades que costuma seguir. A pesquisadora preenchia a tabela de acordo com a resposta do participante. No segundo momento, o paciente respondia novamente sobre as atividades realizadas em um dos últimos sete dias em que esteve internado.

As entrevistas tiveram cerca de uma hora de duração. Aconteceram no leito da enfermaria, onde os pacientes ficaram em quartos individuais. Vale destacar que foi solicitada a retirada do acompanhante durante este período, visando a confidencialidade das respostas e evitando possível constrangimento.

O "Diário de Atividades" é um instrumento que foi criado por Emmel (2012), é estruturado em nove categorias que agrupam diversas atividades. São elas: atividades de vida diária (AVD), atividades de vida prática (AVP), atividades religiosas, atividades profissionais, atividades educacionais, atividades de 
cuidado com outras pessoas, atividades esportivas, atividades de lazer/diversão e comportamentos. Para cada uma das atividades incluídas nessas categorias, o entrevistado respondeu qual o tempo de dedicação que destinava a elas durante o dia; se estava realizando mais alguma atividade simultaneamente; quem mais estava com ele; onde estava; o grau de satisfação em realizar tal atividade; e o grau de significação, ambos com quatro graduações (muito alto, alto, médio e baixo). Tal instrumento ainda não foi validado, porém, conta com pesquisas na área utilizando-o no Brasil com idosos e crianças (Paganelli, 2015; Nunes, 2015).

Foram incluídos nesta pesquisa os pacientes maiores de 18 anos de idade, internados para realização de transplante de células-tronco hematopoéticas, que aceitaram participar da pesquisa. Foram excluídos os que não possuíam data definida para realizar o transplante de células-tronco hematopoéticas, bem como os que desejaram/necessitaram sair da pesquisa por qualquer motivo ou que evoluíram para óbito.

A pesquisa teve início após a aprovação pelo Comitê de Ética em Pesquisa com Seres Humanos da instituição sob parecer $n^{\circ}$ 1.891.198/2017, e a coleta realizada após a assinatura do Termo de Consentimento Livre e Esclarecido. Os dados obtidos foram analisados por meio de análise estatística descritiva simples.

\section{Resultados}

A amostra final foi composta por 18 participantes. Foram excluídos cinco pacientes dos 23 entrevistados inicialmente. A exclusão ocorreu por óbito durante a internação ou pela alta médica antes do período previsto.

No questionário sociodemográfico, foi possível identificar que entre os participantes, $13(72,2 \%)$ foram do sexo masculino e cinco $(27,8 \%)$ do sexo feminino. Em relação ao estado civil, 11(61,1\%) eram solteiros, seis $(33,3 \%)$ casados e um (5,6\%) viúvo. A idade dos participantes variou entre 18 e 66 anos, com média de 42 anos.

As profissões dos participantes foram bastante variadas como, por exemplo, estudantes, motoristas, vigilante e educador social e donas de casa. Quanto à escolaridade, destacam-se sete (38,9\%) que apresentaram Ensino Médio completo e quatro $(22,2 \%)$ Ensino Fundamental completo.

Em relação ao diagnóstico, as leucemias (mieloides e linfoides) foram a maioria com 11 casos $(61,1 \%)$, seguidos de cinco linfomas $(27,8 \%)$, além de um caso $(5,5 \%)$ de anemia aplástica severa e um $(5,5 \%)$ de doença granulomatosa crônica. O tempo de diagnóstico variou entre sete meses e cinco anos, com uma mediana de 12 meses. Ademais, a maioria dos participantes $(83,3 \%)$ realizou somente quimioterapia anteriormente ao transplante.

Os tipos de transplante de células-tronco hematopoéticas realizados foram bastante diversificados, prevalecendo os aparentados, sendo sete (38,8\%), seguidos dos não aparentados e autólogos, ambos com cinco casos $(27,7 \%)$ e um haploidêntico $(5,5 \%)$.

Com relação às complicações apresentadas no período pós-transplante, destaca-se a mucosite, presente em 15 pacientes (83,3\%), a diarreia em 10 (55,5\%) e náu- 
seas e/ou vômito em 9 pacientes (50\%).

O tempo de internação variou entre 12 e 85 dias, com uma mediana de 26,5 dias, sendo a primeira entrevista realizada, em média, 7 dias antes do transplante (D-7), e a segunda, em média, 23 dias após o transplante (D+23).

Com relação às atividades mantidas nas duas entrevistas, observou-se que quatro atividades de vida diária continuaram sendo realizadas por todos os participantes (100\%), como banho/ higiene íntima/uso do vaso, vestuário, alimentação e sono e cochilo. Além dessas, o uso do celular foi mantido por 16 participantes $(88,8 \%)$, seguidas pelas atividades de descanso e conversas, mantidas por 13 dos pacientes (72,2\%); por fim, assistir televisão e dedicar-se às práticas religiosas, mantidas por 12 participantes $(66,6 \%)$.

Sobre o tempo dispendido para realização das atividades nos dois momentos, houve um aumento no tempo das atividades de tratamentos médicos e terapia, descanso e receber visitas, o que pode ser justificado pelo fato da segunda entrevista ser referente a um dia internado. As atividades que apresentaram redução no tempo de realização foram ouvir rádio ou música, alimentação e banho/higiene íntima/uso do vaso.

Dentre os comportamentos mantidos, prevaleceu ficar quieto, reflexivo, citado por nove pacientes $(50 \%)$, seis $(33,3 \%)$ se mantiveram ansiosos, dois (11,1\%) se mantiveram chateados ou deprimidos e um (5,5\%) apresentou choro antes e após o transplante.

Dentre as atividades realizadas antes da internação, 16 participantes (88,8\%) destacaram locomoção e deslocamento, e sete $(38,8 \%)$ passear e sair de casa atividades que não podem ser realizadas pelos pacientes imediatamente após o transplante, devido ao período de internação e isolamento (figura 1). Neste momento, aparecem também atividades que envolvem o domicílio, do qual estão ausentes, como quatro participantes $(22,2 \%)$ apontaram, como, por exemplo, cuidar de crianças, e cinco $(27,7 \%)$ apontaram os serviços domésticos.

Figura 1. Atividades realizadas antes da internação

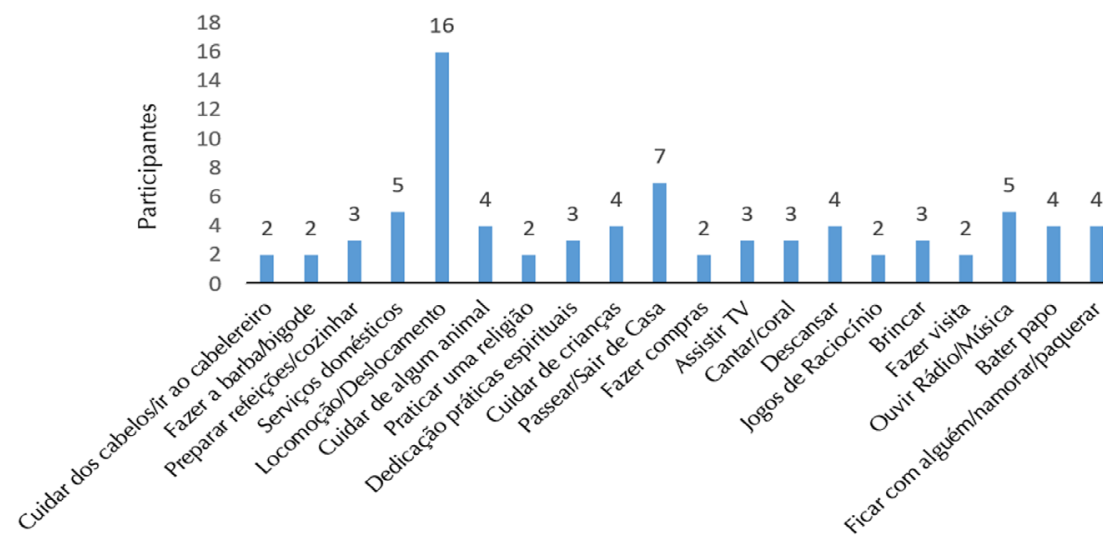

Fonte: Elaboração própria 
Sobre as atividades realizadas durante o período de internação, destacam-se os 14 participantes $(77,7 \%)$ que fizeram tratamentos médicos e terapias, $6(33,3 \%)$ receberam visitas e quatro $(22,2 \%)$ apresentaram comportamentos (como o fato de chorar), três $(16,6 \%)$ ficaram ansiosos e dois $(11,1 \%)$ ficaram chateados/deprimidos (figura 2).

Quando levamos em conta o quanto as atividades realizadas são significativas para quem as realiza, ou seja, o quanto é importante poder realizar aquela atividade, verificamos que, antes da internação, a atividade considerada como a mais significativa (muito alto), foi o trabalho remunerado, assim como nas atividades mais significativas na internação. Em seguida, identifica-se passear/sair de casa, viagem de lazer, tratamentos médicos e terapias e praticar uma religião. Conclui-se, então, que estas atividades não sofreram impacto em sua relevância durante o processo de transplante (figura 3 ).

Figura 2. Atividades realizadas durante a internação

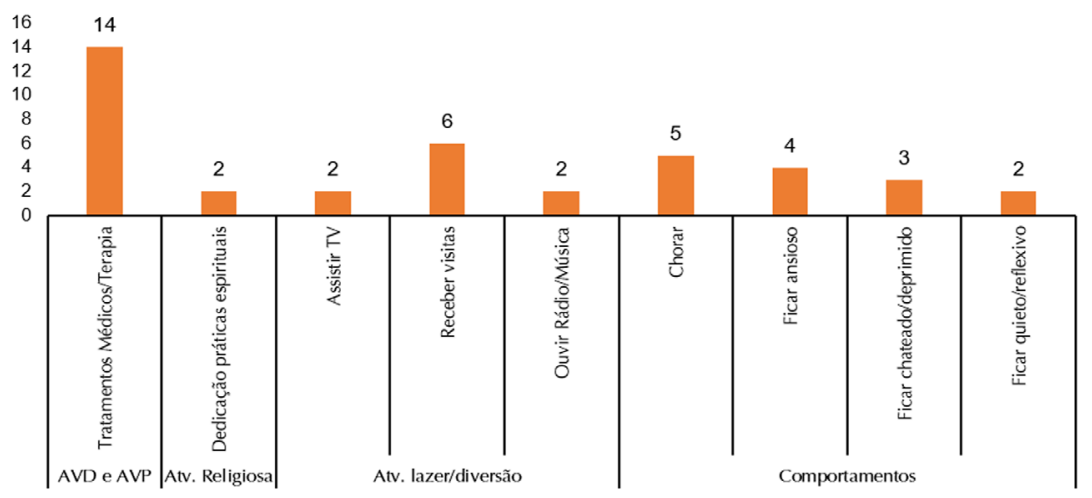

Fonte: Elaboração própria

Figura 3. Comparativo das atividades mais significativas antes e durante a internação

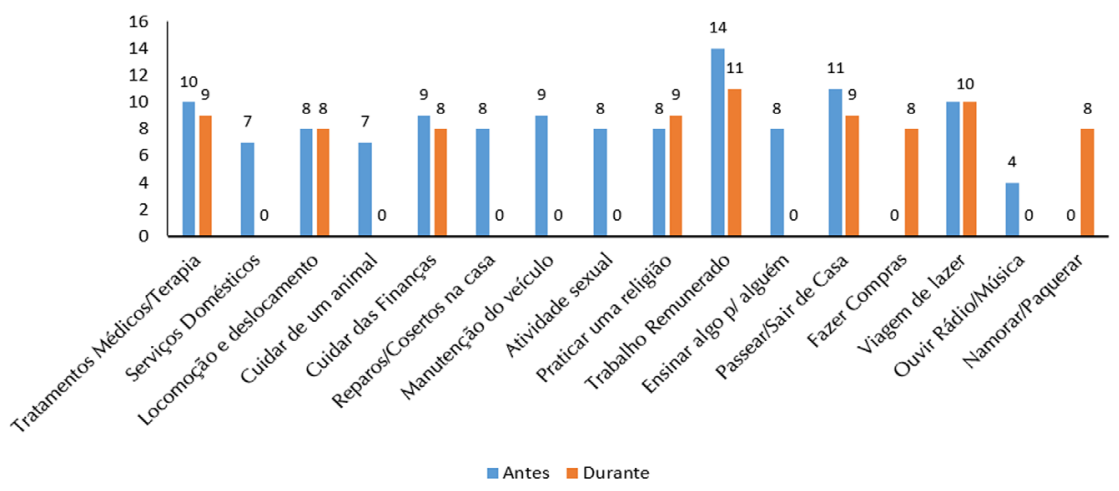

Fonte: Elaboração própria 
Já as atividades de manutenção do veículo, atividade sexual, serviços domésticos, fazer reparos e consertos na casa, ensinar algo para alguém e ouvir música, foram citadas como muito significativas antes da internação. Por outro lado, fazer compras e namorar/ paquerar, que não foram identificadas como muito significativas na primeira entrevista, foram identificadas como tal, na segunda entrevista. Nota-se, também, uma diminuição na quantidade de atividades identificadas como significativas no período de internação.

Ainda com relação às atividades significativas, as que se mantiveram significativas, mas não classificadas como muito alto, foram: dedicação às práticas espirituais, descanso e uso do celular.

Quando levamos em consideração a satisfação em realizar as atividades, evidencia-se uma queda significativa na alimentação (muito alto para médio ou baixo), não acompanhando o significado, que manteve-se alto ou muito alto. Sendo assim, conclui-se que apesar da baixa satisfação, que pode ser relacionada à mucosite e aos episódios de náusea e/ou vômitos, esta atividade continuou sendo importante para os participantes. Em contrapartida, observou-se um aumento (baixo ou médio, para muito alto) na satisfação em relação ao banho/higiene íntima/uso do vaso, enquanto a significação não apresentou mudanças importantes, mantendo-se entre alto e muito alto.

As atividades de dedicação às práticas espirituais e descanso não apresentaram mudanças significativas na satisfação, mantendo-se alto ou muito alto.

\section{Discussão}

Observou-se que a maioria dos participantes desta pesquisa eram do sexo masculino, assim como na pesquisa realizada por Mastropietro (2006). No entanto, esta não é uma constante, visto que Mastropietro, Oliveira-Cardoso, Simões, Voltarelli \& Santos (2010) encontraram uma maioria de pacientes do sexo feminino, o que demonstra que este fator não é homogêneo, uma vez que as patologias atingem ambos os sexos.

O período de transplante de células-tronco hematopoéticas pode ser de grandes mudanças, dentre elas, deixar o trabalho, atividade que se manteve como muito significativa nas duas coletas. Nos achados de Mastropietro et al. (2010), a maioria dos pacientes entrevistados, após o transplante, estavam afastados de seu trabalho, tendo a renda advinda de benefícios sociais.

Assim como no presente estudo, Dias et.al (2012) verificaram que o desempenho do papel ocupacional de trabalhador e o desejo de retomá-lo após o transplante de células-tronco hematopoéticas sofrem alterações durante o processo de transplante. Desta maneira, apesar desta atividade apresentar grande significação para os pacientes, sua retomada pode ser lenta e com obstáculos, como as condições clínicas. Algumas vezes, pode ser carregada de frustrações e restrições, que podem ser físicas, médicas ou psicológicas (Andrade, Castro, Santos \& Soares, 2012). Deve ocorrer de forma gradual e, muitas vezes, adaptada à nova condição e restrições. Assim, faz necessária a presença do terapeuta ocupacional neste processo, que na sua atuação levará em consideração suas capacidades atuais em seu posto de 
trabalho. Em alguns casos, considerará também as mudanças em sua identidade pessoal, após o processo de adoecimento, além da relação com os colegas de trabalho (Lancman \& Ghirardi, 2002).

Atividades como namorar/paquerar e fazer compras, possivelmente eram atividades já realizadas antes da internação, porém adquiriram nova importância, tornando-se mais significativas para alguns participantes. Tal fator pode ser explicado pela ressignificação do cotidiano e novas formas de ver e viver a vida pelos pacientes, após a experiência do transplante de células-tronco hematopoéticas (Andrade et al., 2012).

Algumas atividades que foram citadas, só foram realizadas antes do transplante, como locomoção/deslocamento e passear/sair de casa. Tal fato pode ser justificado pelo isolamento protetivo, necessário devido a imunodepressão intensa dos pacientes, ficando assim predispostos a complicações infecciosas (Nucci \& Maiolino, 2000).

O isolamento também pode ser considerado fator predisponente para $\mathrm{o}$ aparecimento de sintomas, tais como depressão e medo (Oliveira-Cardoso, Mastropietro, Voltarelli \& Santos, 2009). É preciso considerar tal fator, pois, nesta pesquisa, houve relato de choro, ficar chateado/deprimido, ficar ansioso e ficar quieto, reflexivo, na segunda entrevista.

As reações à hospitalização são diferentes em cada paciente, sendo os fatores determinantes ainda desconhecidos. Entretanto, sabe-se que o significado pessoal e subjetivo que a doença física desperta é fundamental, podendo ser associado à personalidade, circunstâncias sociais e pela característica da doença e seu tratamento (Botega, 2006). Desta forma, não foi possível identificar uma constante nos comportamentos apresentados pelos participantes.

O período de internação, principalmente pós-transplante, também é marcado pelas complicações, que podem interferir na realização de algumas atividades como, por exemplo, a alopecia, que impede os pacientes de cuidar dos cabelos/ir ao cabelereiro ou fazer a barba/bigode, fazendo com que estas atividades apareçam somente antes da internação.

Além destas, encontramos a presença de sintomas gastrointestinais e a mucosite, inflamação do trato gastrointestinal, em decorrência de radioterapia ou quimioterapia, que pode afetar a alimentação (Araujo et al, 2015). A mucosite oral atinge cerca de $90 \%$ dos pós-transplantados e sua gravidade dependerá do diagnóstico, regime e doses utilizados no condicionamento (Guedes et al., 2004). Este fator pode ter influenciado na queda da satisfação na realização da atividade de vida diária de alimentação. Porém, tal atividade continuou com alto grau de significação, ou seja, ainda sendo muito importante para os pacientes, mesmo sem conseguir realizá-la.

A justificativa da manutenção da significação pode estar relacionada ao fato de a alimentação não se reduzir à ingestão de nutrientes, estando intimamente ligada às sensações, experiências e lembranças, sendo assim vinculada ao prazer. Em pacientes hospitalizados, a busca pelo prazer está associada à angústia gerada e, em alguns casos, à proximidade com a morte (Corbeau, 2005). 
No pós-transplante imediato, os pacientes estão mais voltados às atividades de autocuidado, como, por exemplo, o gerenciamento medicamentoso e seguir rigorosamente as orientações médicas (Mastropietro, Santos \& Oliveira-Cardoso, 2006). Se naturalmente indivíduos adultos gastam quase $50 \%$ do seu tempo total com atividades de cuidados pessoais (Lopes \& Coelho, 2017), durante a hospitalização, esse tempo torna-se ainda maior, sobrando menos tempo para realização de outras atividades, como pode ser observado no decréscimo da quantidade de atividades realizadas antes e após o transplante, bem como no aparecimento da atividade de tratamento médico/terapia, citada na segunda entrevista.

Pode-se afirmar que o processo de adoecimento e a hospitalização podem causar uma ruptura no cotidiano do indivíduo (De Carlo, Bartalotti \& Palm, 2004). Desta forma, o uso do tempo será diferente para pacientes antes da internação e durante a internação. Justifica-se, então, o aumento do tempo dispendido para atividades, como descanso e receber visitas durante a hospitalização, já observado no presente estudo.

Maior necessidade de descanso pode ser explicada pela fadiga, sintoma frequente em pacientes submetidos ao transplante de células-tronco hematopoéticas, e está associada à quimioterapia e radioterapia, utilizadas no condicionamento (Oliveira-Cardoso et al., 2009). Tal fator, demanda mais descanso, o que se relaciona ao aumento no tempo para realização desta atividade, visto nesta pesquisa.

Já o maior tempo recebendo visitas na internação pode estar associado ao horário diário destinado à visitação na unidade. As visitas têm como objetivo oferecer apoio e conforto ao paciente, favorecendo sua recuperação (Kojo et al, 2004).

Tendo em vista as rupturas sofridas durante este processo, o terapeuta ocupacional contribui para o resgate ou desenvolvimento de habilidades, motiva o paciente a ter uma atitude mais participativa e criativa. Além disso, desenvolve maior independência e autonomia nas atividades desenvolvidas, melhorando a organização do cotidiano hospitalar (Bittencourt \& Santos, 2018).

O presente estudo teve como limitações o instrumento utilizado, ainda não validado, e a amostra pequena e restrita a um único centro transplantador, dificultando a generalização dos resultados. Entretanto, acredita-se que os dados obtidos são de grande relevância para a estruturação de planos e ações terapêuticos ocupacionais, que contribuam para a recuperação e qualidade de vida de pacientes submetidos ao transplante de células-tronco hematopoéticas.

\section{Conclusão}

O transplante de células-tronco hematopoéticas, principalmente no período de internação, ainda não conta com muitas pesquisas de terapeutas ocupacionais no Brasil, ainda mais quando se trata da população adulta.

O processo de hospitalização para realização do transplante de células-tronco hematopoéticas pode causar ao indivíduo uma série de mudanças nas atividades que realiza, tanto em re- 
lação ao tempo que dispende para realização destas, como na satisfação em realizá-las ou em sua significação.

Atividades anteriormente significativas sofrem alterações durante a hospitalização, ou novos significados podem surgir, como visto neste estudo com as atividades de namorar/paquerar e fazer compras.

Além disso, a queda na satisfação, em atividades que se mantiveram significativas, como a alimentação, aponta para a necessidade de um trabalho multiprofissional, visando a integração do cuidado.

Apesar das limitações, os resultados desse estudo mostraram-se relevantes para a elaboração do plano terapêutico ocupacional. Ressalta-se, ainda, a importância da presença do terapeuta ocupacional na equipe multiprofissional, para auxiliar na retomada de atividades significativas, no engajamento de novas atividades ou nas adaptações que possam torná-las mais satisfatórias.

\section{Referências}

American Occupational Therapy Association -AOTA. (2015). Estrutura da prática da terapia ocupacional: domínio \& processo. $3^{a}$ ed. Traduzida. Revista de Terapia Ocupacional da Universidade de São Paulo, 26(ed. esp.), 1-49.

Andrade, A. M., Castro, E. A. B., Santos, K. B. \& Soares, T. C. (2012). A vida após o transplante de medula óssea: implicações para o cotidiano. Cogitare Enfermagem, 17(2), 290-296. DOI: http:// dx.doi.org/10.5380/ce.v17i2.27883
Araújo, S. N. M., Luz, M. H. B. A., da Silva, G. R. F., Andrade, E. M. L. R., Nunes, L. C. C. \& Moura, R. O. (2015). O paciente oncológico com mucosite oral: desafios para o cuidado de enfermagem. Revista Latino-Americana de Enfermagem, 23(2), 267-274 DOI: 10.1590/01041169.0090.2551

Bergkvist, K., Fossum, B., Johansson, U. B., Mattsson, J. \& Larsen, J. (2018). Patients' experiences of different care settings and a new life situation after allogeneic haematopoietic stem cell transplantation. European journal of cancer care, 27(1). DOI: https://doi.org/10.1111/ ecc. 12672

Bittencourt, R. S. \& Santos, D. R. (2018). O terapeuta ocupacional na Unidade de Transplante de Medula Óssea. En: M. M. R. P. De Carlo \& A. M. Kudo (orgs.), Terapia ocupacional em contextos hospitalares e cuidados paliativos (pp. 196204). São Paulo: Editora Payá.

Botega, N. J. (2008). Reação à doença e à hospitalização. En: N.J.Botega, Prática Psiquiátrica no Hospital Geral (pp. 4966). Porto Alegre: Artmed.

Braveman, B., Hunter, E. G., Nicholson, J., Arbesman, M. \& Lieberman, D. (2017). Occupational therapy interventions for adults with cancer. American Journal of Occupational Therapy, 71(5). DOI:10.5014/ajot.2017.715003

Cavalcanti, L. G. A., Paulo, M. A. \& Hany, F. E. S. (2010). A pesquisa piloto de uso do tempo do IBGE 2009/2010. Fazendo Gênero, 9, 1-10. Recuperado de http://www.fazendogenero.ufsc.br/9/ resources/anais/1278295122_ARQUIVO_artigolBGE-APesquisaPilotodeUsodoTempodolBGE2009-2010.pdf 
Corbeau, J. P. (2005). Alimentar-se no hospital: as dimensões ocultas da comensalidade. En A. M. Canesqui. \& R. W. D. Garcia (orgs.), Antropologia e nutrição: um diálogo possível (pp. 227-238). Rio de Janeiro: Editora Fiocruz.

De Carlo, M. M. R. P., Bartalotti, C. C. \& Palm, R. C. M. A Terapia ocupacional em reabilitação física e contextos hospitalares: fundamentos para a prática. En: M. M. R. P. Carlo \& M. C. M. Luzo (Orgs.), Terapia ocupacional: reabilitação física e contextos hospitalares (pp. 3-28). São Paulo: Rocca.

Dias, V. N., Mastropietro, A. P., Oliveira-Cardoso, É. A., \& De Carlo, M. M. R. P. (2012). Transplante de células-tronco hematopoéticas-um estudo controlado sobre papéis ocupacionais. Cadernos de Terapia Ocupacional da UFSCar, 20(2). DOI: http://dx.doi.org/10.4322cto. 2012.016

Doimo, L. A., Derntl, A. M. \& Lago, O. C. (2008). O uso do tempo no cotidiano de mulheres idosas: um método indicador do estilo de vida de grupos populacionais. Ciencia \& Saude Coletiva, 13 (4), 1133-1142. DOI: https://dx.doi.org/10.1590/S141381232008000400009

Emmel, M. L. G. (2012). Ocupação Humana e uso do tempo em atividades significativas ao longo do ciclo da vida: implicações para a qualidade de vida (Projeto de pesquisa).

Fontoura, N., Pinheiro, L., Galiza, M. \& Vasconcelos, M. (2010). Pesquisas de uso do tempo no Brasil: contribuições para formulação de políticas de conciliação entre trabalho, família e vida pessoal. Revista Econômica, 12 (1), 11-46. DOI: https://doi.org/10.22409/economica.12i1.p10

Gomez-Lillo, S. (2003). La ocupación y su significado como factor influyente de la identidad personal. Revista Chilena de Terapia Ocupacional, (3), 43 - 47. DOI: 10.5354/0719-5346.2010.149

Guedes, A. G., Ortega, E. T. T., Meyer, K. L., Vallim, E. T. A., Zavadil, E. T. C. \& Kojo, T.K. (2004). Complicações agudas do transplante de células-tronco hematopoéticas. En E. T. T.Ortega, D. H. Lima, M. P. Veran, T. K. Kojo, \& M. I. Neves, Compêndio de enfermagem em transplante de células-tronco hematopoéticas: rotinas e procedimentos em cuidados essenciais e em complicações (pp.195-226). Curitiba: Editora Maio.

Kojo, T. K., Oliveira, A. M. A., Graciano, A. P. R., da Motta, C. L., Cristoff, C., de Carvalho, D.,... Barão, S. L. (2004). O processo TCTH na unidade de internamento. En: E. T. T. Ortega, D. H. Lima, M. P. Veran, T. K. Kojo \& M. I. Neves, Compêndio de enfermagem em transplante de células-tronco hematopoéticas: rotinas e procedimentos em cuidados essenciais e em complicações (pp.127-166). Curitiba: Editora Maio.

Lancman, S. \& Ghirardi, M.I.G. (2002). Pensando novas práticas em terapia ocupacional, saúde e trabalho. Revista de Terapia Ocupacional da Universidade de São Paulo, 13(2), 44-50. DOI: http:// dx.doi.org/10.11606/issn.2238-6149. v13i $2 \mathrm{p} 44-50$

Lopes, M. \& Coelho, E. (2017). Diferenças e semelhanças entre o uso do tempo das crianças e dos adultos em Portugal. Boletim Sociedade Portuguesa de Educação Física, 0(24-25), 9-31. Recupera- 
do de https://boletim.spef.pt/index.php/ spef/article/view/107

Mastropietro, A. P. (2003). Reconstrução do cotidiano de pacientes submetidos ao transplante de medula óssea: readaptação funcional e reinserção ocupacional (Dissertação de mestrado). Universidade de São Paulo, Brasil.

Mastropietro, A. P., Oliveira-Cardoso, E. A., Simões, B. P., Voltarelli, J. C. \& Santos, M. A. (2010). Relação entre renda, trabalho e qualidade de vida de pacientes submetidos ao transplante de medula óssea. Revista Brasileira de Hematologia e Hemoterapia, 32 (2), 102-107. DOI: https://dx.doi.org/10.15 90S1516-84842010005000035

Mastropietro, A. P., Santos, M. A. \& Oliveira-Cardoso, E. A. (2006). Sobreviventes do transplante de medula óssea: construção do cotidiano. Revista de Terapia Ocupacional da Universidade de São Paulo, 17(2), 64-71. DOI: http:// dx.doi.org/10.11606/issn.2238-6149. v17i2p64-71

Nucci, M. \& Maiolino, A. (2000). Infecções em transplante de medula óssea. Medicina, 33, 279-293. DOI: http:// dx.doi.org/10.11606/issn.2176-7262. v33i3p278-293

Nunes, A. C. (2015). O uso do tempo nas atividades cotidianas e a qualidade de vida de crianças de classe popular (Dissertação de mestrado). Universidade Federal de São Carlos, Brasil.

Oliveira-Cardoso, E. A., Mastropietro, A. P., Voltarelli, J. C. \& Santos, M. A. (2009). Qualidade de vida de sobreviventes do transplante de medula óssea (TMO): um estudo prospectivo. Psicologia: Teo- ria e Pesquisa, 25(4), 621-628. DOI: https://dx.doi.org/10.1590/S0102 37722009000400018

Oliveira-Cardoso, E. A., Voltarelli, J. C., Santos, M.A. \& Mastropietro, A.P. (2005). Intervenção junto à família do paciente com alto risco de morte. Medicina, 38 (1), 63-68. DOI: http:// dx.doi.org/10.11606/issn.2176-7262 . v38i1p63-68

Paganelli, L. O. (2015). O uso do tempo de idosos que participam de grupos para a terceira idade do município de São Carlos (Dissertação de mestrado). Universidade Federal de São Carlos, Brasil.

Pasquini, R. (2004). Fundamentos e biologia do transplante de células hematopoéticas. En M. A. Zago, R.P. Falcão \& R. Pasquini, Hematologia: fundamentos e prática (pp. 913-934). São Paulo: Editora Atheneu.

Paulino, V. U., Paulino, G. C., Puga, G. B. G., \& Bianchin, M. A. (2007). Transplante de médula osea: abordaje de la terapia ocupacional. Revista electrónica de terapia ocupacional Galicia, TOG, (6), 3. Recuperado de: http://psiqu.com/2-14925

Santos, D. D. F. K., Camargo, M. J. G., Santos, D. R., \& Lolatto, G. A. (2017). O uso de estratégias de coping de pacientes adultos submetidos ao transplante de células-tronco hematopoéticas. Revista Ocupación Humana, 17(2). Recuperado de https://www.latinjournal.org/ index.php/roh/article/view/169 